\title{
Experimental and Numerical Study of Free Convection on an Isothermal Downward Cone
}

\author{
M. ASHJAEE, T. YOUSEFI, M. ARZAGHI, M. JARAHI \\ Mechanical Engineering Department \\ University of Tehran \\ Tehran 11365-4563 \\ IRAN
}

\begin{abstract}
The laminar free convection heat transfer from an isothermal downward cone is investigated experimentally and numerically. The experimental investigation is carried out by Mach-Zehnder interferometry technique and the numerical simulation was done by Fluent. The cone tip angle has been kept constant to $45^{\circ}$ and it has been suspended from its base throughout the experiment. This paper focuses on the effect of Rayleigh number variation on the local and average free convection heat transfer coefficient over the conical surface. The local and average Nusselt numbers were determined for the Rayleigh number range of $4.9 \times 10^{5}$ to $1.1 \times 10^{6}$. Also the experiment and the numerical simulation were carried out on a vertical isothermal cylinder of circular cross section in order to compare results with other researchers for the verification of our experimental and numerical results. The significant influence of the upper end surface of the cone, both in the experimental and numerical studies indicated a recirculation region above the upper end surface which affects the local convection heat transfer at the slant trailing edge and causes it to increase. Also a correlation for the calculation of the local Nusselt number over the cone is proposed.
\end{abstract}

Key-Words: Free Convection, Downward Cone, Mach-Zehnder, Interferometry, Vertical Cylinder, Conical Surface

\section{Introduction}

The industrial applications of convection heat transfer from cones could be found in civil engineering, chemical industry (bottom of tanks), and electronics (transistors, resistors, diodes, lamps).

The problem of laminar free convection flow along a vertical faced up or down cone has been treated theoretically in the literature by many researchers since 1953. Merk and Prins [1] found the similarity solution for the case of an isothermal cone whereas Hering and Grosh [2] have obtained a number of similarity solutions for cones with prescribed wall temperatures being a power function of the distance from the apex along the generator. Further results were obtained by Hering [3] and Sparrow and Guinle [4] for small values of Prandtl number (Pr) and by Roy [5] for large values of Prandtl number, respectively. Also, Alamgir [6] has investigated the overall heat transfer from vertical cones using the integral method. Further, Pop and Takhar [7] have studied the compressibility effects in laminar free convection from a vertical cone, while Hossain and Paul [8] have considered the effect of suction and injection when the cone surface is permeable. In all these analytical papers, the boundary-layer thickness is small compared to the local radius of the cone. Also, Lewandowski et al. [9] studied the problem of laminar free convection from an upward cone theoretically. Their work also include experimental results for the average heat transfer rates from the cones with different tip angles, and the two limiting cases of vertical cylinder and circular disc as special cases. In their analytical work, they assumed the parabolic temperature profile in the boundary layer which is applicable only to thin boundary layer flow and should be modified to take into account the effect of thick boundary layer resulting at low Rayleigh numbers. Besides, several mixed convection studies from cones are done by other researchers.

An experimental measurement of mean heat transfer rates from vertical cones has been done by P.H. Oosthuizen and E. Donaldson [10] in which the model has been treated as a lumped object and they have concentrated on finding the average free convection heat transfer coefficient. Natural convection heat transfer from vertical cylinder in the unbounded space has been investigated experimentally and numerically, previously [11].

A literature search reveals that no experimental studies have been carried out for the determination of the local heat transfer coefficients on the surface of downward isothermal cones where the boundary layer thickness is comparable to the diameter of the cone.

The present study focuses on finding the variation of the local and average heat transfer coefficients on the surface of a downward isothermal cone with respect to 
different Rayleigh numbers. The study is carried out experimentally using Mach-Zehnder interferometer and numerically by Fluent.

\section{Experimental Setup}

\subsection{Interferometer}

A Mach-Zehnder Interferometer (MZI) with $100 \mathrm{~mm}$ optics was used in the experimental study. The output of an interferometer is an interference fringe field that is related directly to the variation of the refractive index in the test model. Although restricted to fluids that are transparent, this optical technique can give nonintrusive temperature field measurement without calibration and with no thermal inertia. A basic MZI consists of a light source, two mirrors and two beam splitters. Figure 1 shows a schematic of the interferometer used in the experiment. Beam splitters BS1 and BS2, along with plane mirrors M1 and M2 constituted the basic MZI. Further information about MZI can be found in [27, $28,29]$. The light source which was used is a $100 \mathrm{~mW}$ Helium-Neon laser, $\lambda=632.8 \mathrm{~nm}$.All the MZI components were positioned so that the light beam was incident on each of them at an angle of $30^{\circ}$. This is different from the conventional MZI setting of $45^{\circ}$. The reason for using $30^{\circ}$ is that a wider beam can be obtained with this angle, thereby increasing the field of view. All the interferograms were digitized with a "Panasonic WV-CP410" 1/3" CCD camera with 440000 pixel. To acquire the interferogram the camera was connected to a video recorder through a PC. At each surface temperature we recorded three interferograms. Figure 2 shows one of the images which are recorded by the CCD camera. For quantitative measurement of local free convection heat transfer coefficients over lateral surface of the cone, a reference fringe mode was employed adjusting the mirrors of the interferometer so that the fringes outside the thermal boundary layer extend perpendicular to the axis of symmetry. The reason for applying reference fringe mode in regions with low local heat transfer rates is that there is insufficient number of isotherms near the surface, and it is desirable to measure the surface temperature gradient directly from the interference fringe field, where the reference fringe shifts represents the variation of refractive index in the flow field.

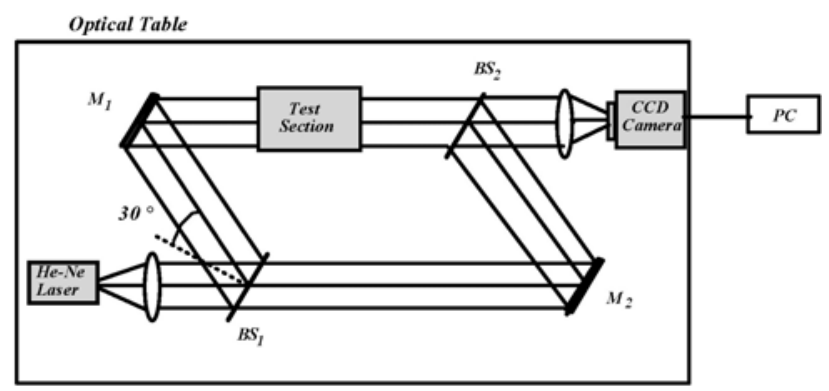

Fig.1 Plan view of the Mach-Zehnder interferometer

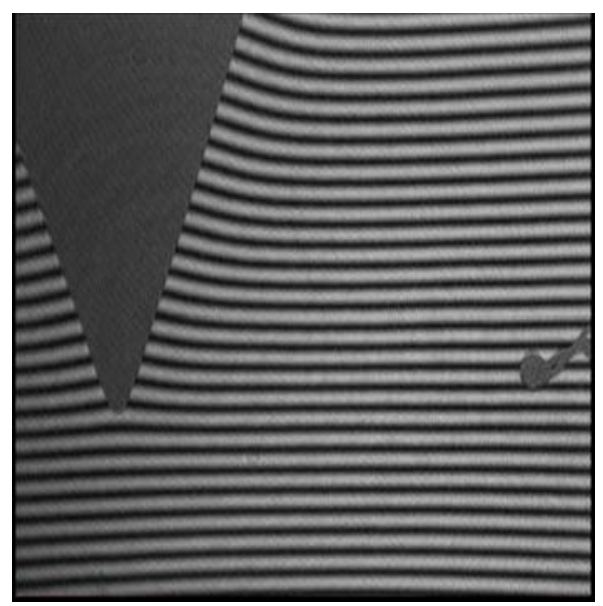

Fig.2 A sample Interferogram of the vertical cone at finite fringe mode

\subsection{Experiment Test Section}

The tested vertical cylinder and cone were made of aluminum and are shown schematically in figures 3 and 4 respectively. The cone base diameter is $43 \mathrm{~mm}$ with the tip angle of $45^{\circ}$. The length and diameter of the vertical cylinder was chosen $12.7 \mathrm{~mm}$ and $92 \mathrm{~mm}$ respectively in order to provide results comparable to the previous work [26, 32] for verifying the method of the experimental data reduction which has been used. Also wooden end caps with thermal conductivity of $0.05 \mathrm{~W} / \mathrm{mK}$ were installed on the bases of both the cylinder and the cone to minimize the end effect.

The surface of both objects was highly polished to assure smoothness and the reduction of the radiation heat transfer. Both objects, the cylinder and the cone, were hollow; therefore a heater could be placed inside them in order to achieve different surface temperature. The vertical cylinder was filled with magnesium oxide powder. By passing electricity through its heater by a $20 \mathrm{~V}-2 \mathrm{~A}$ power supply and considering relatively thick walled aluminum tube, we could achieve constant cylinder surface temperature. For heating the cone thin strip sheet heaters were wrapped on the surface of a 

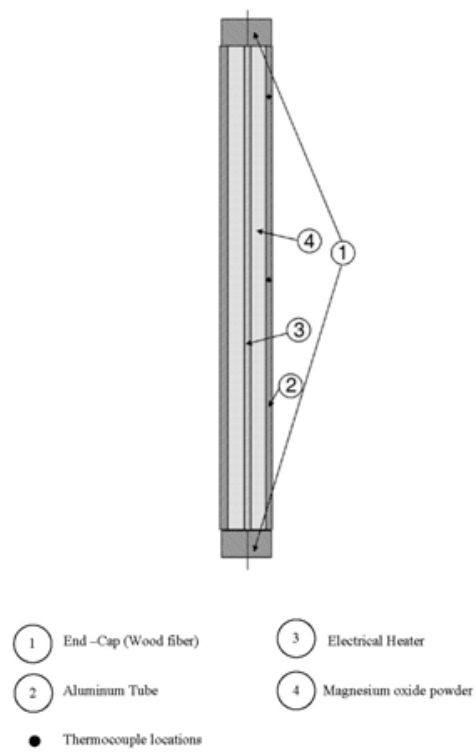

Fig.3 Internal arrangement of the cylinder and thermocouple locations

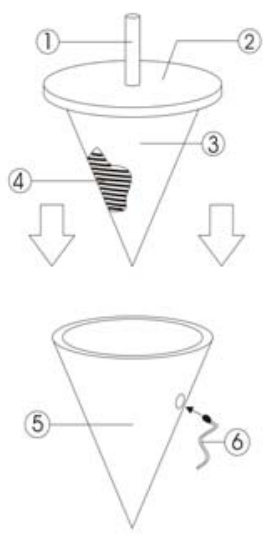

(1) Suspension Wooden Rod

2. Wooden End Cap

Wooden Cone Wrapped with

(3) the fire Resistance Cloth

Fig4. Internal arrangement of the cone and thermocouple locations

wooden cone with a cap assembly. The distances between these strips were kept small enough to achieve uniform surface temperature. The wooden wrapped cone is then covered by a fire resistant cloth to prevent the electrical contact between the heaters and the hollow aluminum cone. Finally, the wooden cone with the wrapped heater covered by the cloth was placed inside the aluminum hollow cone. By passing electricity through the heater by a $30 \mathrm{~V}-10 \mathrm{~A}$ power supply and considering relatively thick walled aluminum conical surface, we could achieve uniform and constant surface temperature over the surface of the cone. The variation of temperature on both surfaces was below $0.3^{\circ} \mathrm{C}$, which is reasonable for constant surface temperature assumption on both models. The electrical power supplied to the heater was controlled by a variable transformer, therefore obtaining different surface temperatures. The supplied electrical power was measured via a digital wattmeter. The local surface temperatures of the heated cylinder and cone were measured via type $\mathrm{K}$ thermocouples, embedded in the surface by means of pinned-junction method. The thermocouples location on the cylinder was $50 \mathrm{~mm}$ far from one end, and on the cone it has been mounted at $15 \mathrm{~mm}$ from its base. Two other thermocouples of the same type were used to measure the ambient and the reference temperatures for data reduction. All the temperatures were monitored continuously by a "TESTO 177" four channel data logger which was connected to a PC. The thermocouples and the data logger set were calibrated before installation. In order to calibrate the thermocouples at two data points, the junction readings were recorded in the mixture of icewater and boiling water at the laboratory pressure. The laboratory pressure and relative humidity were recorded during all the experiments. The cone and the cylinder were suspended from their bases such that their vertical axis along the gravity was perpendicular to the laser beam.

In order to eliminate the effect of any other air disturbances on the experimental test section, the entire setup was housed within a top open Plexiglas box of dimensions $0.5 \times 0.5 \times 0.5 \mathrm{~m}$. Also the box had two side openings for the laser beam passage.

\section{Experimental Data Reduction Method}

The 3-D nature of the temperature field around both objects, the vertical cylinder and the downward cone, arise a need for a quantitative method other than 2-D conventional data reduction method [12] to investigate the distribution of change of refractive index in the radial direction.

The direction of the laser beam which passes through the cone thermal boundary layer is parallel to the $X$ axis, figure 5, which is being perpendicular to the cone axis $Z$.In this case we have a variation of index of refraction in the radial direction because of the presence of a disturbance due to buoyancy induced flow over the cone and the optical path is no longer uniform. The fringe shift calculation for axisymmetric objects is fully explained by Ostrovsky [15], Hauf \& Grigull [12], and Vest [14]. The reference fringe mode has been employed for measurement of temperature gradient over lateral surface of the cone. 
In infinite fringe mode the information fringe shift is gained in a discontinuous manner; i.e., $\delta=0,0.5,1, \ldots$

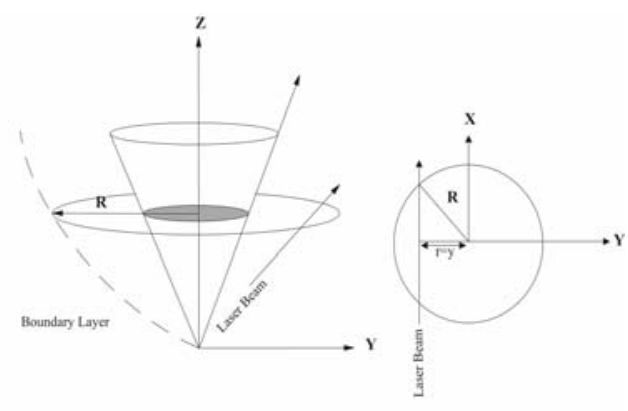

Fig.5 Disturbed phase in a plane of the $Z=$ constant

The fringe shifts $\delta$ is the difference, in wavelengths, of the optical paths of two light beams, one of which passes through undisturbed and the other through a disturbed medium. Under the assumption that $n-n_{\infty}=K\left(\rho-\rho_{\infty}\right)$, this difference is given by [15]:

$\delta(y, z)=\frac{K}{\lambda} \int_{x_{1}}^{x_{2}}\left[\rho(x, y, z)-\rho_{\infty}\right] d x$

In a plane $\mathrm{z}=$ constant let the disturbance be bounded by the circle $r=\mathrm{R}$. Then we see from the figure 5 that along any light ray with $\mathrm{y}=$ constant, Eq. (1) becomes

$\delta\left(y^{2}\right)=\frac{K}{\lambda} \int_{y^{2}}^{R^{2}} \frac{\left[\rho\left(r^{2}\right)-\rho_{\infty}\right] d r^{2}}{\left(r^{2}-y^{2}\right)^{1 / 2}}$

This is an Abel -type integral equation, with the solution:

$$
\rho\left(y^{2}\right)-\rho_{\infty}=-\frac{\lambda}{\pi K} \int_{y^{2}}^{R^{2}} \frac{\left[d \delta\left(r^{2}\right) / d r^{2}\right] d r^{2}}{\left(r^{2}-y^{2}\right)^{1 / 2}}
$$

To summarize, several methods are available for the analysis of the above integral. In the usual reduction process, classical methods, to obtain the density distribution in a plane of constant $\mathrm{z}$ the equation can be approximated by a finite summation involving the discrete values of $\delta$ along the corresponding line of constant $\mathrm{z}$ in the interferogram. These classical methods have been explained by Ostrovsky [15], Hauf \& Grigull [12], and Vest [14]. All such methods are numerical approximations of equation (2) or (3). The axisymmetric distribution is divided into a number of concentric annular zones, and integration is replaced with summation. Thus, the task of finding the radial distribution of $\rho\left(y^{2}\right)$ consists of solving a system of algebraic equations. Since Eq. (3) involves a derivative of $\delta$, there are inherent dangers in such an approximation. The problem can be alleviated, however, by applying a differentiation scheme to the values of $\delta$ and integrate the function fitted to $d \delta\left(r^{2}\right) / d r^{2}$. If a proper method of differentiation were applied, these functions are suitably wellbehaved and satisfy Eqs. (2) and (3). Another solution is to fit curve to $\delta\left(r^{2}\right)$ then differentiate the curve and calculate the integral. This procedure also needs a great accuracy in curve fitting; whereas a little deviation from the actual function of $\delta$ will result in an incorrect temperature profile on the surface. Finally, there are several transform methods which have been referred to by Vest [[13].

Considering a transform method introduced by James W. Bradley [16] for obtaining the density distribution from an axisymmetric interferogram, let $t=1-y^{2} / R^{2}$. From what is known of the properties of the fringe shift, it is reasonable to assume that for constant $\mathrm{z}, \delta / t^{0.5}$ can be expanded in a power series in $t$ :

$$
\delta(t)=t^{0.5} \sum_{n=0}^{\infty} B_{n} t^{n}
$$

Using a Laplace transform, Bradley [16] assumed that for a constant $\mathrm{z}, \delta / t^{0.5}$ can be represented adequately over the unit interval by, say, a cubic in $\mathrm{t}$ :

$$
\delta / t^{0.5}=B_{0}+B_{1} t+B_{2} t^{2}+B_{3} t^{3}
$$

We determine the coefficients by a least squares fit of the fringe shift values and obtain $\rho$ at once from Eq.(5):

$$
\rho-\rho_{\infty}=\frac{1}{2 A}\left[B_{0}\left(\frac{3 B_{1}}{2}\right) t+\left(\frac{15 B_{2}}{8}\right) t^{2}+\left(\frac{35 B_{3}}{16}\right) t^{3}\right]
$$

where $A=\frac{K R}{\lambda}$ and finally the temperature profile can be found as follows:

$$
T=\left(\frac{1}{T_{\infty}}-\frac{3 \cdot R_{0} \cdot\left(\rho-\rho_{\infty}\right)}{2 \cdot P_{\infty}}\right)^{(-1)}
$$

A MATLAB code has been developed to calculate the complete temperature distribution in the radial direction around the cone in seven imaginary horizontal planes which are perpendicular to the axis of symmetry of the cone. The ineterferograms were read and the fringe shifts where calculated in $0.018 \mathrm{~mm}$ radial intervals within the test area by the method described in [[13].

The Laplace transform was then applied to the available fringe shifts in the same way that was outlined above to determine temperature distribution. Consequently, the free convection heat transfer coefficient over the lateral surface is determined 
locally by reading the temperature gradient at the surface as follow:

$$
h_{\xi}=-\left.k \frac{d T}{d r}\right|_{r=0} \cdot \frac{1}{\left(T_{w}-T_{\infty}\right)}
$$

where $h_{\xi}$ is the local heat transfer coefficient, and $\xi$ is the distance measured from the tip of the cone and the cylinder base along their lateral surfaces and $k$ is the air thermal conductivity. Therefore:

$$
N u_{\xi}=\frac{h_{\xi} \xi}{k}=-\left.\frac{\xi}{\left(T_{w}-T_{\infty}\right)} \cdot \frac{d T}{d r}\right|_{r=0}
$$

Where $\xi$ is the characteristic length which has been the local distance from the cone tip.

The average free convection heat transfer coefficient, $\overline{h_{L}}$, is calculated from:

$\overline{h_{L}}=\frac{1}{L} \int_{0}^{L} h_{\xi} \cdot d \xi$

where $L$ is the cone slant length.

The thermophysical properties of the air were estimated at film temperature, which is defined as:

$T_{f}=\frac{T_{w}+T_{\infty}}{2}$

For the calculation domain only one half of the meridian plane of the cone is taken because of the symmetry.

In order to check the accuracy of the experiment and the method of data reduction the experiment also was carried out on a vertical isothermal cylinder and the local free convection coefficient were compared with the numerical study and the previous works [26, 32]. Figure 6 shows these results which are in good agreement with the numerical simulation and the previous works.

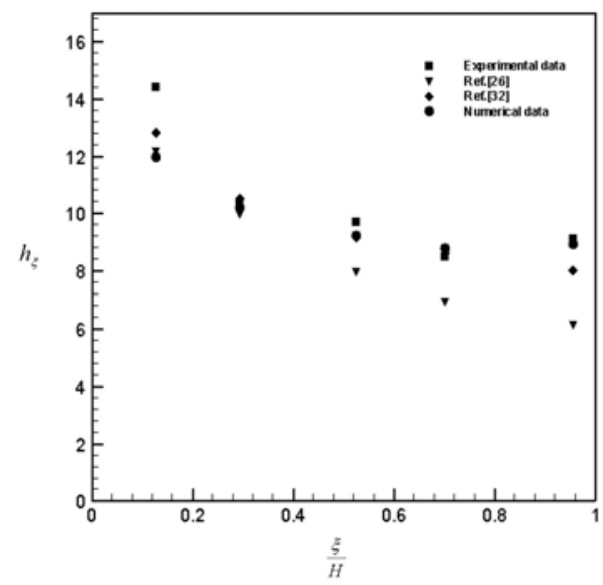

Fig. 6 Local heat transfer coefficient versus dimensionless length for cylinder

\section{Numerical Simulation}

The physical model under consideration is depicted in figure 7. A cone with tip angle $\theta$ and slant length $L$ suspended vertically downward. The Numerical calculations are carried out using cylindrical coordinate system $(z, r)$ with its origin located at the tip of the cone as indicated in the figure. The laminar, steady state, two dimensional and segregated model was used from Fluent.

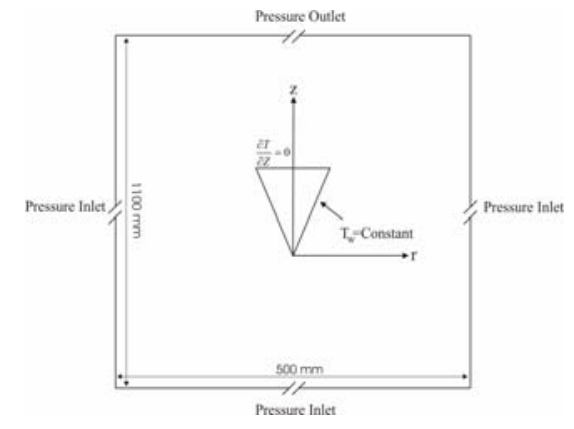

Fig. 7 Physical model and its boundary conditions

By employing the Boussinesq approximation for the buoyancy force, the governing equations are given by:

Continuity: $\quad \frac{\partial}{\partial z}(r u)+\frac{\partial}{\partial r}(r v)=0$

Momentum: $\quad \frac{\partial}{\partial z}\left(u^{2}-v \frac{\partial u}{\partial z}\right)+\frac{1}{r} \frac{\partial}{\partial r} r\left(v u-v \frac{\partial u}{\partial r}\right)=$

$$
-\frac{1}{\rho} \frac{\partial}{\partial z}(P+\rho g z)+g \beta\left(T-T_{\infty}\right)
$$

$\frac{\partial}{\partial z}\left(u v-v \frac{\partial v}{\partial z}\right)+\frac{1}{r} \frac{\partial}{\partial r} r\left(v^{2}-v \frac{\partial v}{\partial r}\right)=$

$-\frac{1}{\rho} \frac{\partial}{\partial r}(P+\rho g z)-\frac{v v}{r^{2}}$

Energy: $\frac{\partial}{\partial z}\left(u T-\frac{v}{p r} \frac{\partial T}{\partial z}\right)+\frac{1}{r} \frac{\partial}{\partial r} r\left(v T-\frac{v}{p r} \frac{\partial T}{\partial r}\right)=0$

In the above equations, $u$ and $v$ are the velocity components in the $z$ and $r$ directions while $T$ and $P$ denote the temperature and pressure.

The cone was placed in an extended space for the numerical solution domain. At left, right and bottom sides of this extended zone we applied pressure inlet condition. The pressure outlet condition was applied at the top side. For the cone lateral and base surfaces the constant temperature and adiabatic wall have been applied respectively. These boundary conditions are shown in figure 7.The problem was solved for different cone surface temperatures. An unstructured grid was applied for this model. On the cone surface the boundary layer mesh was used. The numerical 
results which are in excellent agreement with the experimental data point are shown in figures 8,9, 10 .

\section{Results and Discussion}

Figures 8, 9, 10 indicate the variation of the local convection heat transfer coefficient from the tip of the cone along the lateral surface for a range of Rayleigh numbers between $4.9 \times 10^{5}$ to $1.1 \times 10^{6}$. All the figures show a decreasing trend up to approximately $5 \mathrm{~mm}$ from the base due to the growth of thermal boundary layer.

The significant influence of the base of the cone, both in the experimental and numerical studies, indicates a recirculation region above the cone base which affects the convection heat transfer at the trailing edge and causes it to increase. This effect can be confirmed with the presentation of the velocity field around the cone obtained from the numerical simulation which is indicated in figure 11. The same phenomenon has been observed by Koyama et al. [18].

A correlation has been obtained for the local Nusselt number variation along $\xi$ for different values of the Rayleigh number:

$$
N u_{\xi} / R a_{\xi}{ }^{0.7}=0.001125(\xi / L)^{-1.5}
$$

This correlation is based on the both experimental and numerical data points which are in excellent agreement with each other, as shown in figure 12 .

Also an average Nusselt number, $\overline{N u_{L}}=\frac{\overline{h_{L}} L}{K}$, based on the Eq.(10) has been obtained as following:

$$
\overline{N u_{L}}=0.000703 R a_{L}^{0.7}
$$

Fig. 8 Local heat transfer coefficient versus dimensionless length for cone at $R a=7.9 \times 10^{5}$

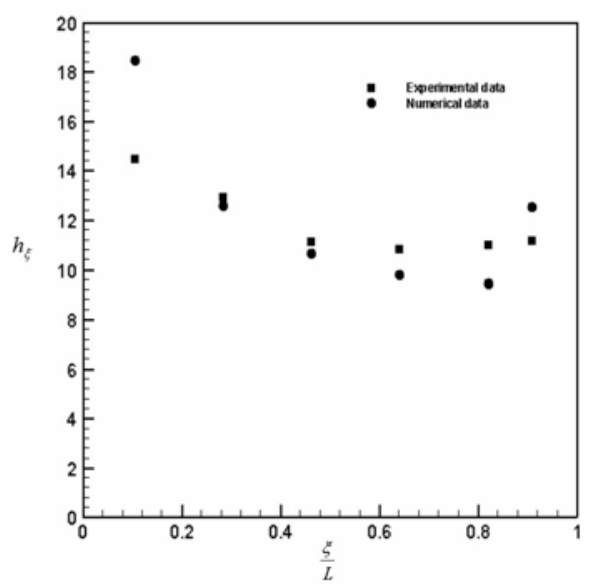

Fig. 9 Local heat transfer coefficient versus dimensionless length for cone at $R a=8.7 \times 10^{5}$

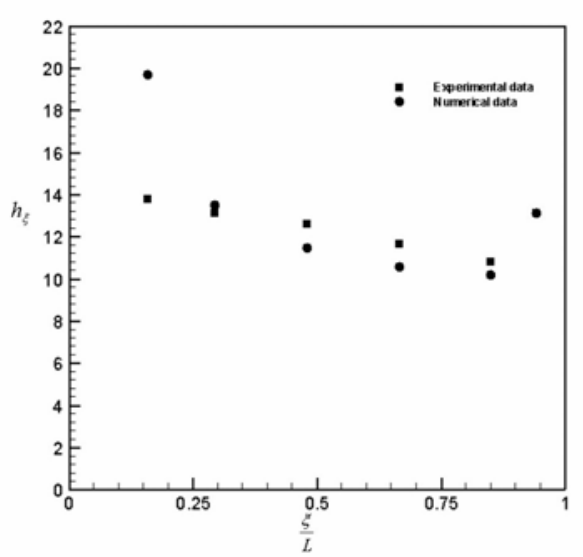

Fig. 10 Local heat transfer coefficient versus dimensionless length for cone at $R a=9.9 \times 10^{5}$

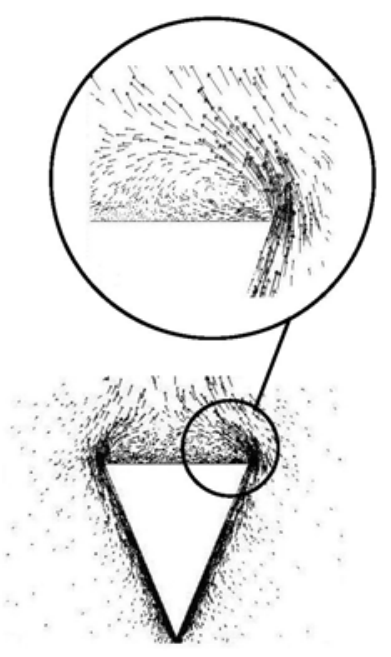

Fig.11 Effect of trailing edge on velocity vector 


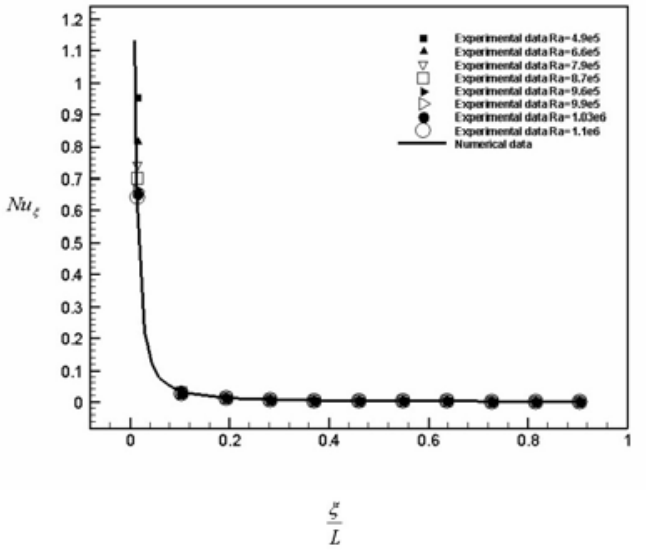

Fig. 12 Local Nusselt number versus dimensionless length

\section{Conclusions}

The laminar free convection from an isothermal downward cone of circular cross section was studied both experimentally and numerically. Experiment was carried out using Mach-Zehnder interferometer. The purpose of the study was to determine the effect of the variations of Rayleigh numbers on the Nusselt number. Correlations have been obtained for local and average Nusselt numbers. The effect of the base of the cone on the heat transfer characteristics was studied and it was indicated a recirculation region above the cone base which affects the convection heat transfer at the trailing edge and causes it to increase.

\section{References}

[1] J.H. Merk, and J.A. Prins, Thermal convection in laminar boundary layer, Applied Scientific Research, vol. 4, pp. 10-24, 1953.

[2] R.G. Hering and R.J. Grosh, Laminar free convection from a non-isothermal cone, Int. J. of Heat and Mass Transfer, vol.5, pp. 1059-1068, 1962.

[3] R.G. Hering, Laminar free convection from a nonisothermal cone, Int. J. of Heat and Mass Transfer, vol. 8,pp. 1059-1068, 1965.

[4] E.M. Sparrow, and L.D.F. Guinle, Deviation from classical free convection boundary layer theory at low Prandtl numbers, Int. J. of Heat and Mass Transfer, vol. 11,pp. 1403-1415, 1968.

[5] S. Roy, Free convection from a vertical cone at high Prandtl numbers, ASME J. of Heat Transfer, vol.96, pp.115-117, 1974.

[6] M. Alamgir, Overall heat transfer from vertical cones in laminar free convection: an approximate method, ASME J. of Heat Transfer, vol. 101, pp. 1746, 1989.
[7] I. Pop, and H.S. Takhar, Compressibility effects in laminar free convection from a vertical cone, Applied Scientific Research, vol. 78, pp. 1-82, 1991.

[8] M.A. Hossain, and S.C. Paul, Free convection from a vertical permeable circular cone with non-uniform surface temperature, Acta Mechanica, vol. 151, pp. 103-114, 2001.

[9] W.M. Lewandowski, S. Szymański, P. Kubski, E. Radziemska, H. Bieszk and T. Wilczewski, Natural convective heat transfer from isothermal conic, Int. J. of Heat and Mass Transfer, vol.42, Issue 10, pp. 18951907, 1999.

[10] P.H. Oosthuizen and E. Donaldson, Free Convective Heat Transfer from Vertical Cones, Transactions of the ASME, pp. 3[19]-331, 1972.

[11] T. Choi, An Interferometric and numerical study of free-, forced-, and mixed convection heat transfer from heated vertical cylinders, Ph.D. thesis, University of Wisconsin-Madison, U.S., 1985.

[12] W. Hauf, , and U. Grigull, Optical Methods in Heat Transfer, In Advances in Heat Transfer, vol. 6, pp. 133-366, Academic Press, New York, 1970.

[[13] E.R.G. Eckert., and R.J. Goldstein, Measurements in Heat Transfer, $2^{\text {nd }}$ ed. McGraw-Hill, New York, 1972.

[14] M. Charles Vest., Holographic Interferometry, John Wiley \& sons, 1979.

[15] Yu.I. Ostrovsky, M.M. Butusov and G.V. Ostrovskaya Interferometry by Holography, SpringerVerlag Berlin Heidelberg, New York, 1980.

[16] J.W. Bradley, Density Determination from Axisymmetric Inteferograms, AIAA, vol. 6, pp. 1190$1192,1968$.

[17] John H. Lienhard IV, and John H. Lienhard V, A Heat Transfer Text Book, $3^{\text {rd }}$ ed. , Phlogiston Press, Cambridge, Massachusetts, 2002.

[18] H. Koyama, A. Nakayama, S. Ohsawa and H. Yamada, Theoretical and experimental study of free convection from a vertical frustum of a cone of a finite length, Int. J. of Heat and Mass Transfer, vol. 28, Issue 5, pp. 969-976, 1985.

[[19]] D. Naylor, Recent developments in the measurement of convective heat transfer rates by laser interferometry, Int. J. Heat and Fluid Flow, vol. 24, Issue 3, pp. 345-355, 2003.

[20] F.N. Lin, and B.T. Chao, Laminar Free Convection Over Two Dimensional and Axisymmetric Bodies of Arbitrary Contour, ASME J. Heat Transfer, vol. 96, pp. 435-442, 1974. 